\title{
High-throughput CRISPR-mediated 3D enrichment platform for functional interrogation of chemotherapeutic resistance
}

\author{
Taraka Sai Pavan Grandhi ${ }^{1}$, Jeremy To $^{2}$, Angelica Mendiola ${ }^{2}$, Fabio Luna ${ }^{2}$, Whitney \\ Barnes $^{2}$, John Walker ${ }^{2}$, Rita Moran ${ }^{2}$, Robbin Newlin², Loren Miraglia ${ }^{2}$, Anthony Orth ${ }^{2}$, \\ and Shane Horman ${ }^{2}$
}

${ }^{1}$ Arizona State University

${ }^{2}$ Genomics Institute of the Novartis Research Foundation

November 9, 2020

\begin{abstract}
Cancer is a disease of somatic mutations. These cellular mutations compete to dominate their microenvironment and dictate the disease outcome. While a therapeutic approach to target specific driver mutations helps to manage the disease, subsequent molecular evolution of tumor cells threatens to overtake therapeutic progress. There is need for rapid, high-throughput, unbiased in-vitro discovery screening platforms that capture the native complexities of the tumor and rapidly identifiy mutations that confer chemotherapeutic drug resistance.Taking the example of CDK4/6 inhibitor (CDK4/6i) class of drugs, we show that the pooled in-vitro CRISPR screening platform enables rapid discovery of drug resistance mutations in a 3D setting. Gene edited cancer cell clones assembled into an organotypic multicellular tumor spheroid (MCTS), exposed to CDK4/6i caused selection and enrichment of the most drug resistant phenotype in a 3D setting, detectable by next gen sequencing after a span of 28 days. The platform was sufficiently sensitive to enrich for even a single drug resistant cell within a large, 2500-cell, drug-responsive complex 3D tumor spheroid. The genome-wide 3D CRISPR-mediated knockout screen $(>18,000$ genes $)$ identified several genes whose disruptions conferred resistance to CDK4/6i. Further, multiple novel candidate genes were identified as top hits only in the microphysiological 3D enrichment assay platform and not the conventional 2D assays. Taken together, these findings suggest that including phenotypic 3D resistance profiling in decision trees could improve discovery and reconfirmation of drug resistance mechanisms and afford a platform for exploring non-cell autonomous interactions, selection pressures, and clonal competition.
\end{abstract}

\section{Hosted file}

SH_High throughput CRISPR mediated 3D enrichment platform for anti-cancer drug resistance discovery.pdf available at https://authorea.com/users/374308/articles/491873-high-throughput-crisprmediated-3d-enrichment-platform-for-functional-interrogation-of-chemotherapeuticresistance 
A.
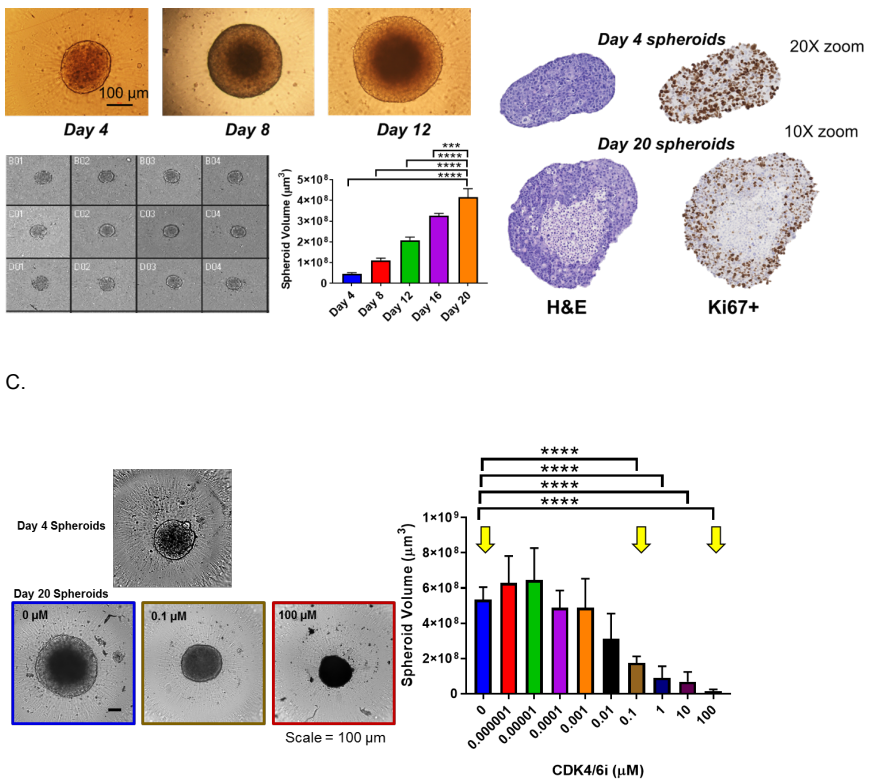

D.

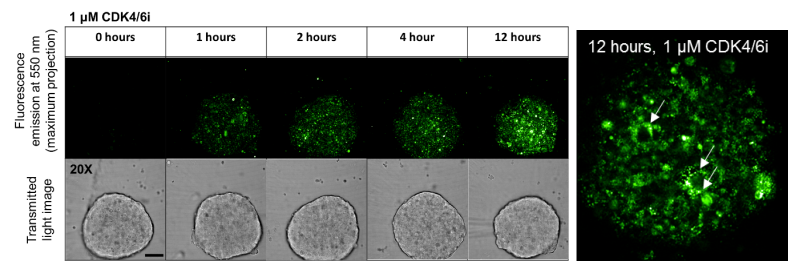


A.

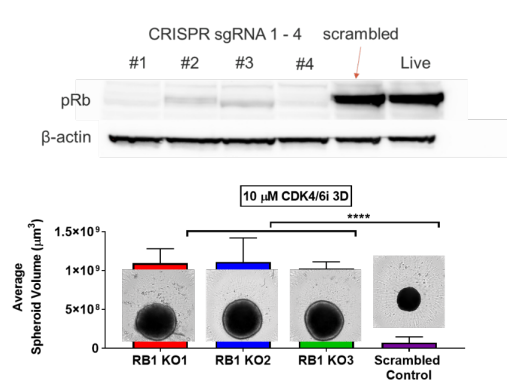

C

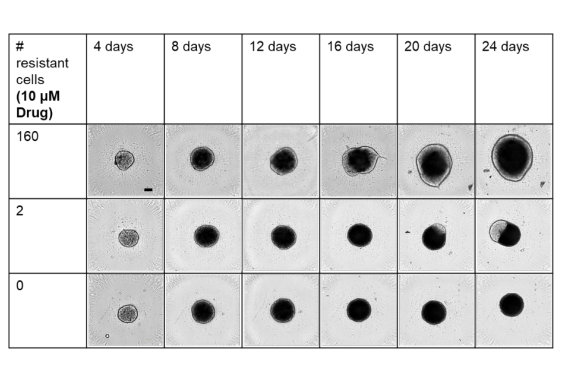

D

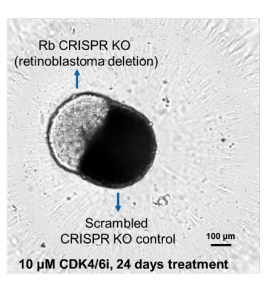

B

E

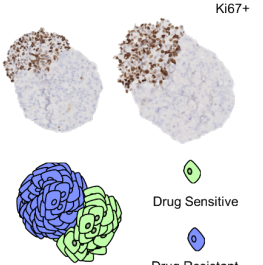

Drug Resistant
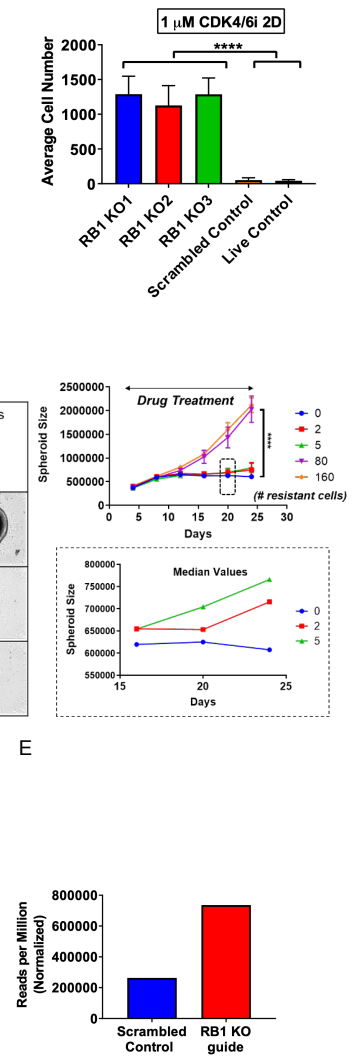

\section{Hosted file}

Fig3.pdf available at https://authorea.com/users/374308/articles/491873-high-throughputcrispr-mediated-3d-enrichment-platform-for-functional-interrogation-of-chemotherapeuticresistance

\section{Hosted file}

Fig4.pdf available at https://authorea.com/users/374308/articles/491873-high-throughputcrispr-mediated-3d-enrichment-platform-for-functional-interrogation-of-chemotherapeuticresistance 

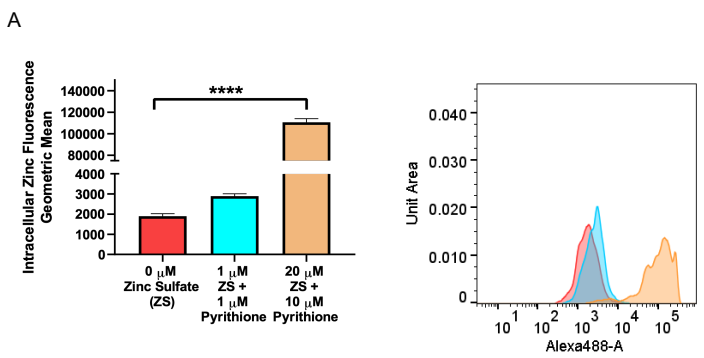

B
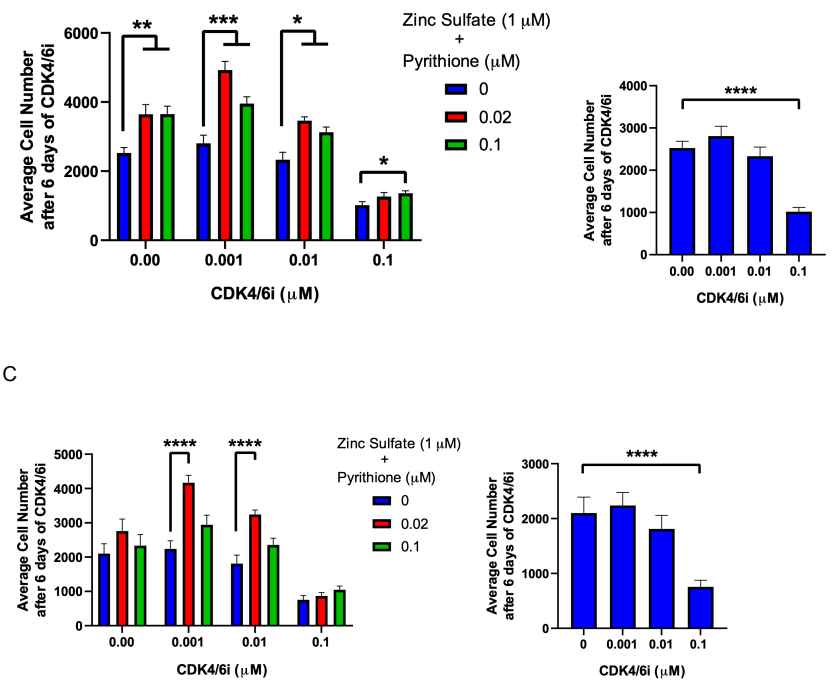